\title{
High content image-based cytometry as a tool for nuclear fingerprinting
}

\author{
$\underline{\text { W.H. De Vos }}{ }^{1}$, B. Dieriks ${ }^{1}$, G. Joss ${ }^{2}$, and P. Van Oostveldt ${ }^{1}$ \\ 1. Department of Molecular Biotechnology, University of Ghent, Coupure links 653, \\ 9000 Ghent, Belgium \\ 2. Department of Biological Sciences, Macquarie University, North Ryde, NSW 2109, \\ Sydney, Australia
}

winnok.devos@ugent.be

Keywords: cytometry, high content screening, segmentation

Cytomics aims at understanding the functional relationships between cellular phenotypes (cytome) and metabolic pathways (proteome) that result from a combination of genetically defined mechanisms (genome) and environmental conditions $[1,2]$. Although flow-cytometry is able to measure the optical properties of single cells at a rate of $>1000$ cells per minute it has a limited capability of mapping individual events. To accurately quantify (sub-) cellular characteristics within a natural context there is a fast-growing need for image-based cytometry. Images, obtained with fluorescence microscopy, provide the exact information on signal intensity, location and distribution of specific molecules within intact cell systems (tissue or monolayers) and allow for investigating cellular properties in relation to the cell-ecological context [3].

Previously, we have developed a cytometric approach for scoring DNA lesion endpoints in confocal images of murine fibroblasts [3]. We now present a generalized approach for multivariate phenotypic profiling of individual nuclei using automated fluorescence mosaic microscopy and optimized digital image processing tools. An indefinite number of fields, z-slices and channels can be analyzed; the only prerequisite is the presence of a nuclear counterstain, which is used for the generation of masks. To anticipate for erroneous segmentation of clustered nuclei in dense cell cultures we implemented an iterative conditional segmentation (ics) algorithm that uses both morphological and intensity information from the image (Figure 1). The method makes use of a priori knowledge about the size and shape of nuclei in stringent feedback selection of correctly segmented nuclei. Depending on the degree of clustering, segmentation performance varies between $95 \%$ and $100 \%$. Complete analysis of nuclei and subnuclear features for a region of 25 images of 1000x1000 pixels, 3 z-slices and 3 channels only takes $\sim 3$ minutes or $\sim 0.7 \mathrm{sec} /$ nucleus. Our method is insensitive to scaling, illumination heterogeneity and variability or non-uniformity of staining.

We have successfully applied our system in cell cycle analysis, scoring of transfection efficiency and assessment of (localized) DNA damage in response to genotoxic stress and ionizing radiation. 
1. T. Bernas, G. Gregori, E. Asem and J. Robinson, Molecular \& Cellular Proteomics 5 (2006), p. 2.

2. G. Herrera, L. Diaz, A. Martinez-Romero, A. Gomes, E. Villamon, R. Callaghan and J. O'connor, Toxicology In Vitro 21 (2007), p. 176

3. G. Valet, J. Leary and A. Tarnok, Cytometry A 59 (2004), p. 167.

4. P. Baert, G. Meesen, S. De Schynkel, A. Poffijn and P. Van Oostveldt, Micron 36 (2005), p. 321.

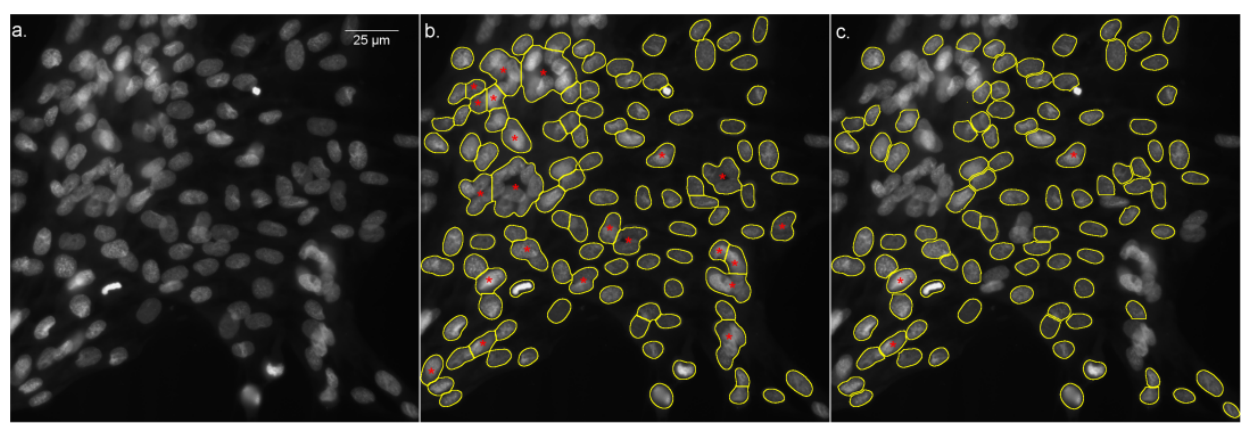

Figure 1. Improved segmentation of dense nuclear images using the ics-procedure. a. Original image of DAPI counterstained human fibroblast nuclei. b. Outlines of general segmentation result superimposed onto the original image. c. Outlines of iterative conditional segmentation superimposed onto the original image. Incorrectly segmented nuclei are marked $(*)$. 